\title{
Neural infrastructure for incremental interpretation: Interfacing sound, meaning and constraint
}

Yuxing Fang, Bingjiang Lyu, Benedict Vassileiou, Kamen Tsvetanov, Lorraine K. Tyler, William D. Marslen-Wilson Centre for Speech, Language and the Brain, Dept of Psychology, University of Cambridge (yf292@cam.ac.uk,wdm10@cam.ac.uk)

The remarkable speed and immediacy of human speech comprehension depends on the dynamic integration of acoustic-phonetic cues from the speech input with multi-dimensional contextual constraints to enable the early recognition of the words being heard and the integration of lexical meaning into the listener's incremental interpretation of the current utterance. This research probes the poorly understood neurocomputational infrastructure that supports these core early ingrative processes. We aim

Identify the critical brain regions involved;

Determine how different cues and constraints are neurally represented,

Measure the precise timing with which these different inputs are integrated.

Investigate the directionality and the content of connectivity processes across the network.

To address these questions we combine source-localized EMEG measures of realRepresentational Similarity Analysis (RSA) and Grainger Causal Analysis (GCA) measures of connectivity with a network approach based on ICA and Lasso rescesson. The methods are applied to an existing source-reconstructed EMEG data set of short sentences of the form 'The elderly man ate the apple" (Lyu et al 2019), focusing on the first $250 \mathrm{~ms}$ after direct object onset (e.g. 'apple'), leading up to word Identification-point (IP).

First (Section 1) we use an ICA whole-brain data-driven approach to decompose

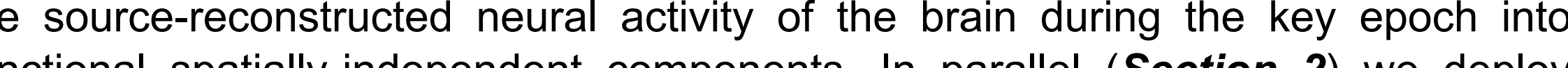
ASR and NLP models of linguistic form and content in order to identify (Section 3) any ICA components that are sensitive to the knowledge-types involved in early integration and to determine (Section 4) the timing of different types of model fit across the epoch. We then (Section 5) use Lasso regression to decompose the selected network into major subcomponents (frontal and temporal) and then compute GCA connectivity between these components. Finally (Sections 6 \& 7) we relate connectivity strength to phonetic and semantic models to probe the neurocomputational content of these connections.

(1) ICA decomposition into candidate networks

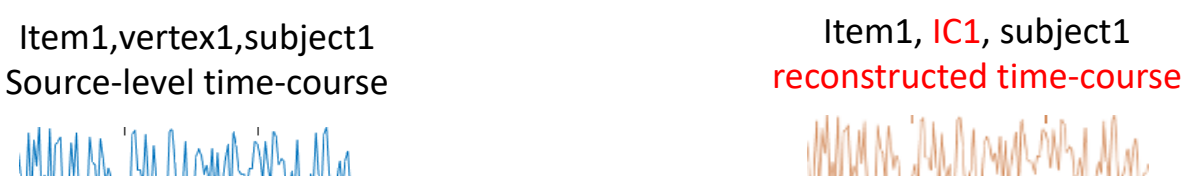

$$
\begin{aligned}
& \text { Mmandind }
\end{aligned}
$$

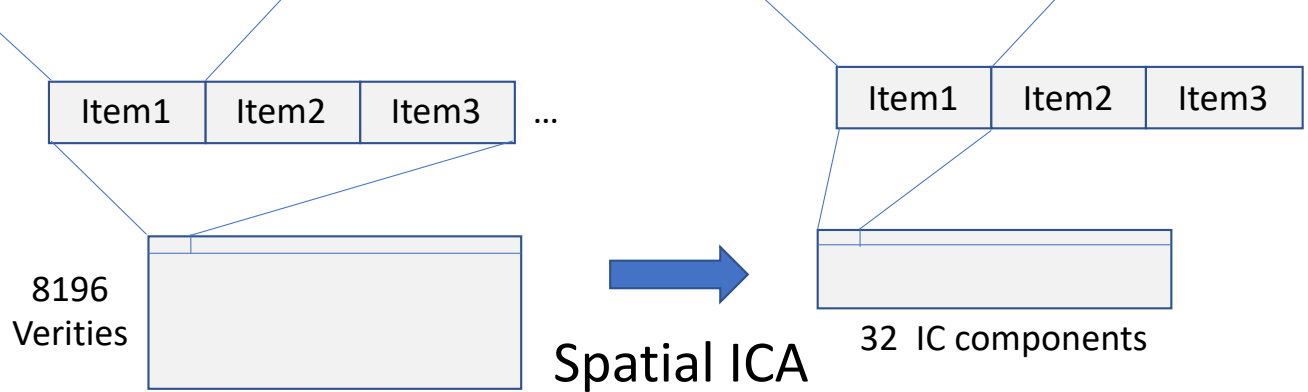

To detect independent networks when processing speech, we first concatenated the source-localized signals from each item and extracted 32 independent components using ICA. To perform RSA analyses on these components, we re-

(2) Computational models for network selection and analysis

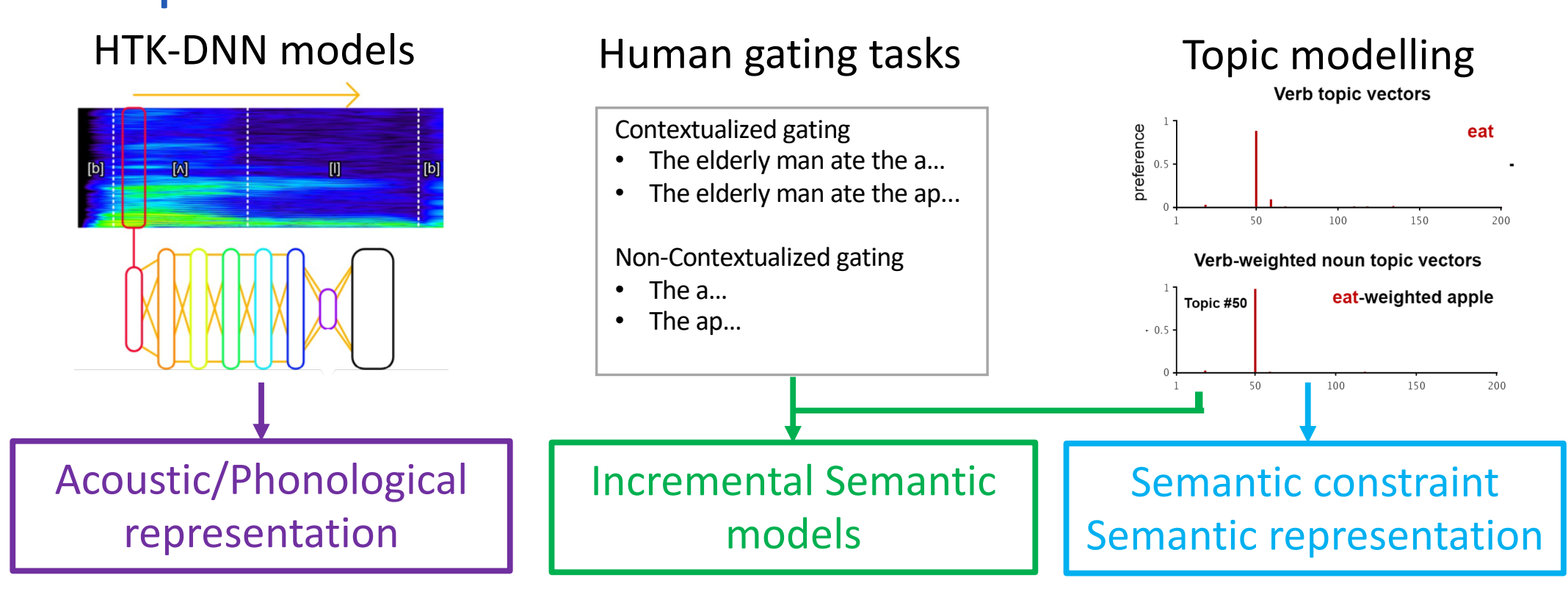

The acoustic-phonetic/phonological properties of the speech input and releva semantic factors are derived from separate ASR and NLP models. Incrementa semantic interpretation itself is captured by models of the semantic properties of the evolving word-initial cohort (estimated from gating studies) at different time-
points (Gate1: 0-50ms, Gate2: 0-100ms, Gate3: 0-150ms) after word onset.

(3) Identifying networks related to integration of sound, meaning, and context a range of component numbers (illustrated
The ICA process, segmenting brain activity on the basis of shared temporal coherence generates multiple potential candidates. For here for $n=32$ ) we used RSA to test each component for its sensitivity to the relevant model types.

Only the network labelled IC24 showed fit to the semantic models - most importantly to the incremental semantic models - as well as to the acoustic-phonetic and phonological models

- The IC24 network is strongly left lateralised and implicates processing regions primarily in IFG and in posterior temporal cortex, including $\mathrm{HG}$, STG and MTG

IC24 is mirrored by a parallel RH network IC16, with similar fronto-temporal loadings. This component only showed fit to acousticphonetic and phonological models
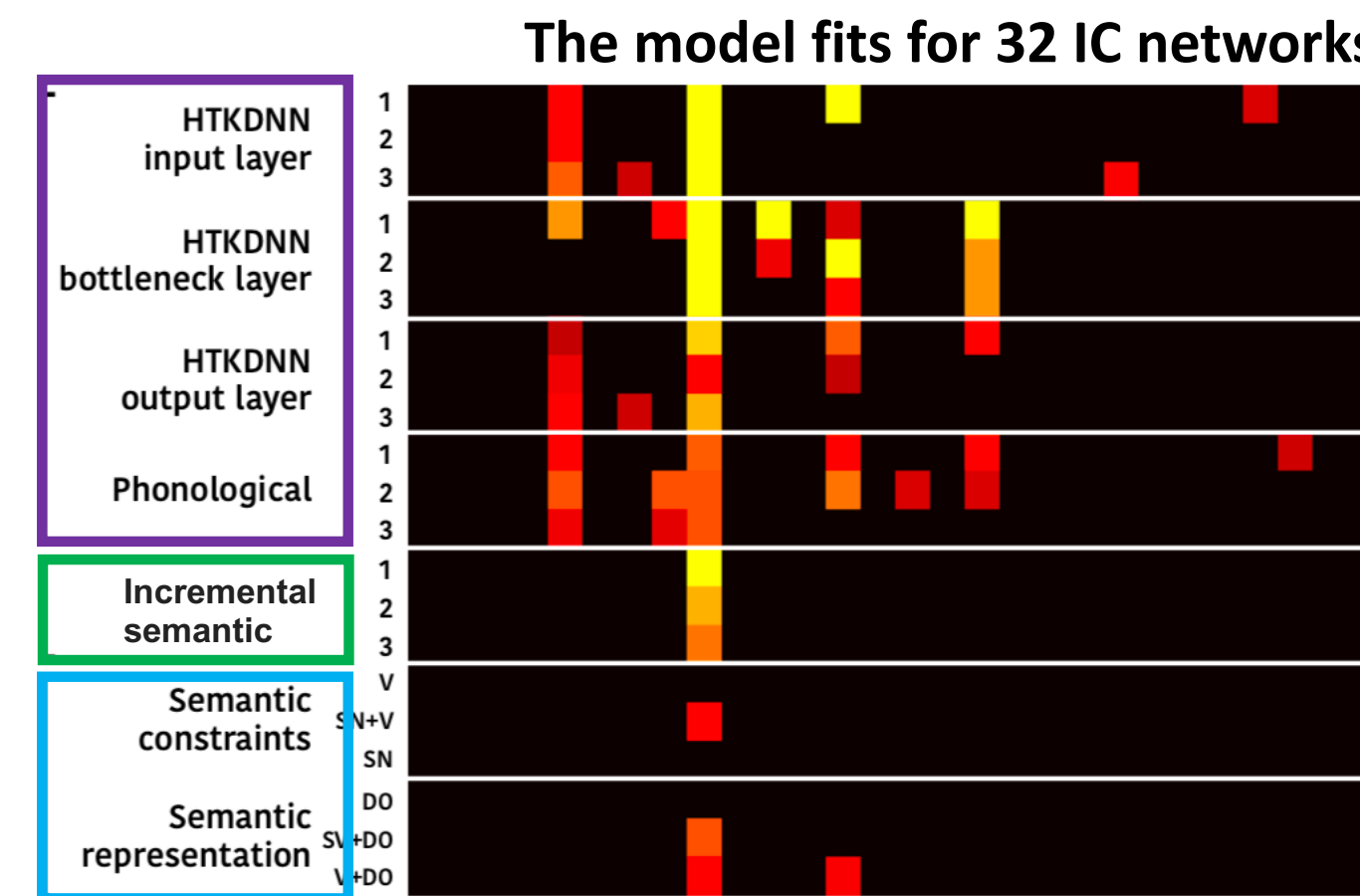

(4) Temporal structure of IC24 model fits

A key question here is the timing with which incremental semantic models reflecting the word being heard start to emerge as a function of ch input combined with contextual constraints.

- Onset of model fit (at $140 \mathrm{~ms}$ ) for the uncontextualized incremental model is delayed relative to contextualized models (at $98 \mathrm{~ms}$ )

Neither model shows model fit from word onset.

Model fit for both terminat identified.

(5) ICA network decomposition and GCA connectivity

To understand the inner structure of IC24, we used a datadriven method to determine the optimal segmentation of weights of IC 24 can be representents. The spatial ICA of the sub-components (networks). The combination was solved by lasso regression while minimizing the number of temporal network.

The directional connectivity between these sub-networks networks was assessed using a pattern-based GCA. Firs we built data RDMs based on the time-varying IC signals from each network. We then calculated the correlation (d) between the RDMs from a certain time point in Region $A$ (a) ,dt=-60ms) and Region $B(b, d t=0 \mathrm{~ms}$, partialling out the contribution to Region $B$ of its own previous state. The resulting GCA-connectivity pattern captures the significant results from testing all dt combinations (from -2 to $-60 \mathrm{~ms}$ ) sub-components. This procedure picked out two components - an IFG-based frontal network and an STG/MTG based
CSLB CAMBRIDGE
(6) Informational content of network connectivity To explore what kind of information is transferred across the IC24 5) to predict the modelfits (sections 3 \& 4) of the whole network over time. Significant model fit is interpreted as evidence about the informational role of the observed connectivity pattern

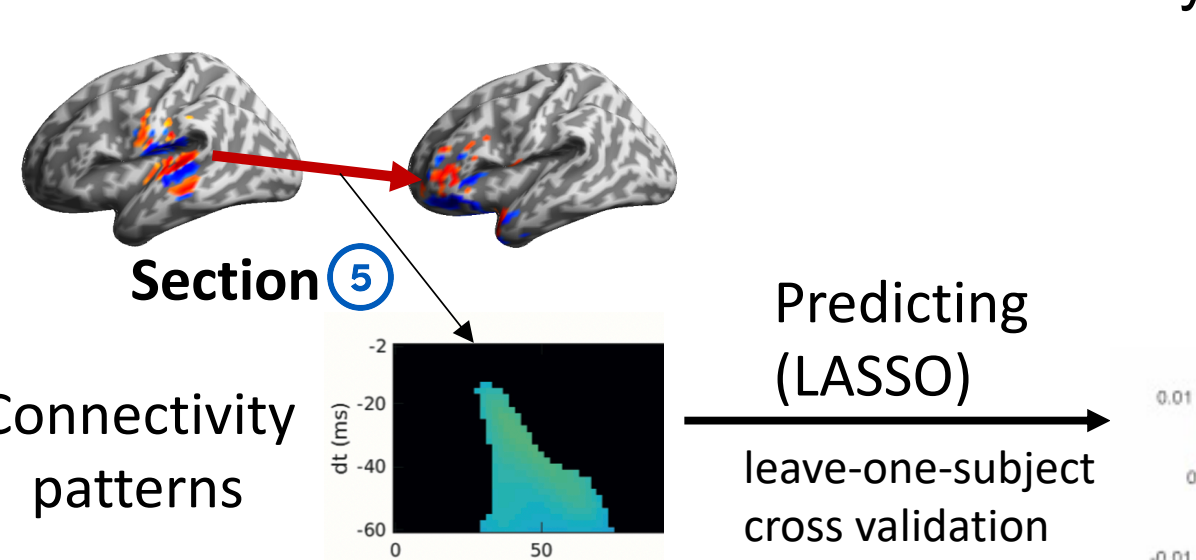

IC24

Sections (3) \& (4)

patterns

of model-fi

Applying these tests to the connectivity patterns between the two main subnetworks, we found that over the first $200 \mathrm{~ms}$ from word onset both acoustic-phonetic content and semantic content were

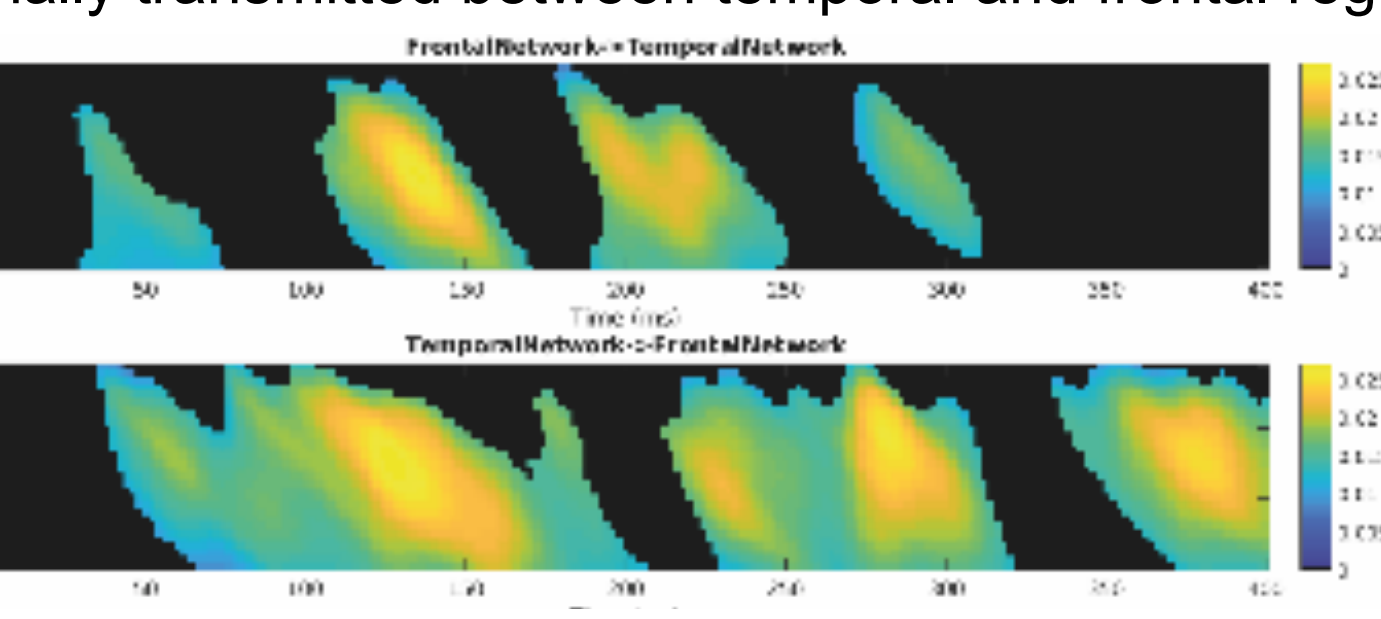

In a second set of analyses we extracted from the frontal

4 component two subsets correspondina to BA 47 and BA 45 .
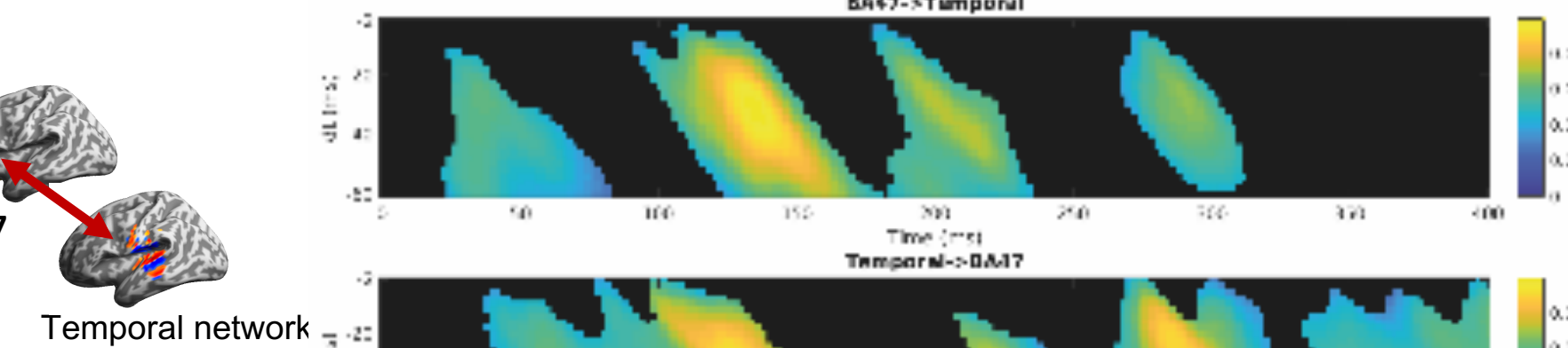

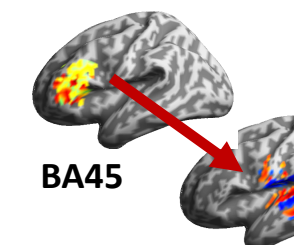

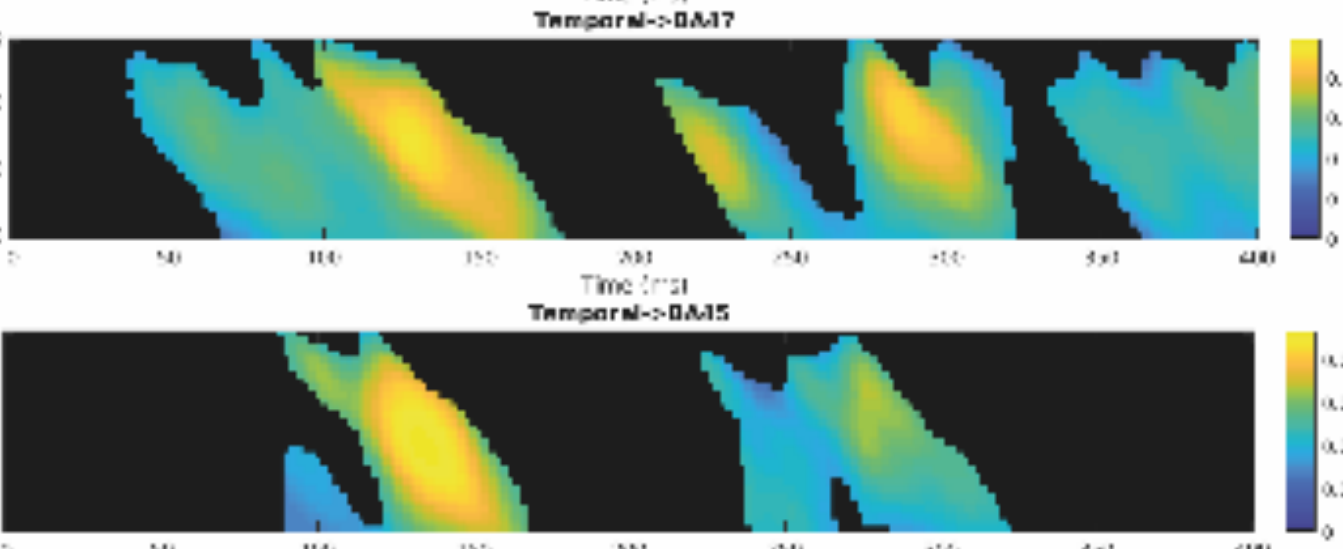

Here we see more differentiated effects. Strong bidirectional connectivity involving BA47 primarily links to semantic models, with very early outflow from BA47. Phonological information plays a minor and later role. BA45 vertices, in contrast, only show connectivity outward to temporal regions, starting relatively later and with model fit strongly dominated by acoustic-phonetic and phonological models

Overview

- Model-based RSA evaluation of ICA components uniquely identifies a LH fronto-temporal network that integrates acoustic-phonetic cues and meaning in the first $200 \mathrm{~ms}$ after word onset in sentential context - Integration of acoustic-phonetic cues with contextual constraints is not visible at word-onset, suggesting that bottom-up constraints are necessary to set the representational geometry of an analysis space

The RH mirror component shows strong sensitivity to acousticphonetic and phonological models, but no systematic response to any semantic models. This indicates a quallative diference in the incremental interpretation of speech

Innovative (but preliminary) analyses of the information content of different roles for ventral and more dorsal regions of IFG 